\title{
Modulating antibacterial properties using
} nanotechnology

\begin{abstract}
"So far it is clear that even an apparently weak physical stimulus (i.e., a nanoscale variation in surface topography or the presence of a very small amount of Ag NPs) may cause important reduction of bacteria adhesion."
\end{abstract}

\section{KEYWORDS: antibacterial " nanocomposites " nanotopography}

Solid materials entering into contact with living systems may bring with them infections, which are caused by microorganisms that first adhere to the surface and then start to colonize and form biofilms [1,2]. This is the case, for instance, for implantable medical devices, such as intravascular catheters, pacemakers, ventricular devices, voice prostheses, dental and orthopedic implants. Conversely, soft materials made of entangled fibers (e.g., paper, tissues and nonwoven fabrics) may originate similar problems in different environments, such as food packaging, dermal contact and surgery. From the material science point of view, the strategies needed to overcome the growth of bacterial colonies change dramatically depending on whether the material has a continuous surface or it consists of entangled fibers.

Highly controlled surface nanotopography is the key method to avoid bacteria adhesion in solid materials having proper surfaces, whereas encapsulation of the fibers into metal/polymer nanocomposites seems to be a highly efficient way to make entangled materials safe.

The development of such innovative nanotechnologies is somewhat at the early stage and requires extensive interdisciplinary studies to elucidate how the physicochemical properties of materials can regulate the responses of biological entities [3,4], the central question concerning the understanding of the complex molecular mechanisms occurring at the interface between nanostructures and living matter.

\section{Solid materials}

For this class of materials, surface nanoroughness is found to influence the biological response of bacteria in terms of cell morphology and variation of the gene expression and proteomic profile. This has recently been demonstrated in a very systematic way [5] by studying the Escherichia coli response to the nanotopography of gold surfaces whose nanoscale roughness was controlled with extremely high precision $(\sim 5 \mathrm{~nm})$ in the range of 20 to $100 \mathrm{~nm}$ by a wet chemical method termed spontaneous galvanic displacement reaction. Bacteria grown on nanorough surfaces exhibit as a main macroscopic difference the absence of type 1 fimbriae compared with those grown on flat surfaces (average roughness $R_{a}<1 \mathrm{~nm}$ ). The fimbriae are long and thread-like adhesive organelles ( 500 per cell) that enable bacteria to target and colonize specific host tissues [6] and to form biofilms [7]. The lack of fimbriae occurs already for nanoroughness values $\mathrm{R}_{\mathrm{a}} \sim 20 \mathrm{~nm}$, suggesting that a surface topographical perturbation of the order of $10^{-4}$ of the bacterium volume is sufficient to induce a strong response in bacterial cells.

\section{"The understanding of the bacterial response and adaptation to nanostructures ... are the key issues to enable the development of nanosurfaces with active, smart and programmable antibacterial properties..."}

At the molecular level, genetic studies indicate that nanoroughness leads to a loss of expression of the bacteria virulence factors with unexpected sensitivity even to mesoscopic (few tens of nanometers) topographic changes. Real-time quantitative PCR shows that the nanoroughness affects the genetic switching of the fimbrial operone. In fact, $E$. coli growing on to the reference flat surfaces have the fimbrial operone in the 'ON' orientation (thus permitting the transcription of all fimbrial subunits), whereas those growing onto nanorough surfaces switch the fimbrial operone into the 'OFF' orientation, preventing the transcription of the fimbrial subunits [8].

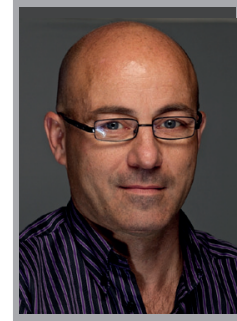

Roberto Cingolani

Author for correspondence: Istituto Italiano di Tecnologia - Via Morego 30, Genova, Italy roberto.cingolani@iit.it

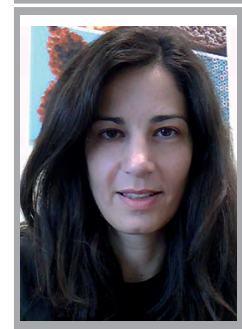

Athanassia Athanassiou Istituto Italliano di Tecnologia - Via Morego 30, Genova, Italy

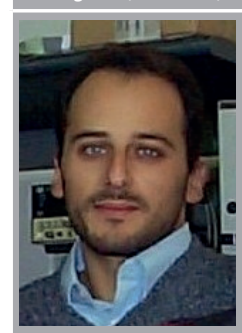

Pier Paolo Pompa Istituto Italiano di Tecnologia, Center for Bio-Molecular Nanotechnologies @UniLe-Via Barsanti, Arnesano (Lecce), Italy 
Nanotopography disorder affects even the proteome of the E. coli. Comparing the proteomic profiling of bacteria grown onto flat and nanorough substrates by 2D differential in gel electrophoresis and mass spectrometry, different expression patterns of proteins involved in biosynthesis, peptide transport, metabolic pathway and DNA repair system were found [5]. In particular, the proteomic studies reveal that bacteria on nanorough surfaces undergo general stress processes, with a consequent overexpression of enzymes involved in the protection of DNA, amino acid synthesis, energy production, regulation and rearrangement of the external membrane. At the same time, a pronounced downregulation of transport proteins and enzymes related to DNA synthesis was detected as a possible defense mechanism of E. coli to avoid damages during DNA synthesis while adhering onto nanorough surfaces.

\section{Fibrous materials}

In the case of materials made from entangled fibers a different approach has to be pursued in order to introduce antibacterial properties in the fiber network. Due to the intrinsic random micro- or nano-roughness of their surface, nanopatterning methods cannot be applied to these materials. In this case postproduction processes to encapsulate the fibers into coatings with antibacterial activity have to be developed $[9,10]$. The individual coverage of each fiber, instead of the formation of an overall coating of the entangled fiber ensemble, is expected to have enhanced antibacterial activity due to the highly increased surface area exposed. This can be accomplished in any fibrous material by solution casting or by dip coating techniques, both being suitable for large area applications. The best antibacterial properties are obtained by wetting the fibrous material with ethylcyanoacrylate (ECA) monomer solutions containing silver nanoparticles (Ag NPs) with a diameter of approximately $10 \mathrm{~nm}[9,101]$. Immediately after solvent evaporation, ECA starts cross-linking around the fibers under ambient conditions due to naturally occurring surface hydroxyl groups and/or adsorbed moisture, encapsulating them with a polymer shell (the Ag NPs/ECA nanocomposite) whose desired antibacterial functionality is ultimately provided by the ion release from the $\mathrm{Ag}$ NPs. The best efficiency of the method is accomplished by nucleating the Ag NPs directly into the ECA monomer solutions, using laser ablation [10] of a solid Ag target. Remarkably, concentrations of $\mathrm{Ag}$ NPs as small as $0.01 \mathrm{wt} \%$ in ECA are enough to provide the treated materials with excellent antibacterial properties. The antibacterial effect of Ag NPs/ECA nanocomposite has been demonstrated on synthetic fibers and natural cellulose fibers, in other words paper, which is the most common prototype of entangled cellulose fibers. The bacterial adhesion and growth (E. coli) were analyzed and compared after immersion of treated and untreated fibrous sheets in the bacterial culture medium. A large decrease in the number of bacterial colonies was found in the Ag NPs/ECA treated fibers compared with the reference untreated sheets. As mentioned above, such antibacterial behavior is ascribed to the effect of $\mathrm{Ag}^{+}$ions released by the Ag NPs dispersed in the polyethylcyanoacrylate cladding of the fibers. $\mathrm{Ag}^{+}$ ions, in fact, are well known to interact with thiol groups of proteins, resulting in the inactivation of respiratory enzymes, and production of reactive oxygen species [11] that prevent DNA replication and affect the structure and permeability of cell membranes [12].

\section{Conclusion}

The exploitation of nanotechnology to modulate antibacterial properties of materials is still at the early stage, but is very promising. So far it is clear that even an apparently weak physical stimulus (i.e., a nanoscale variation in surface topography or the presence of a very small amount of Ag NPs) may cause important reduction of bacteria adhesion. In addition to the physicochemical nanomodifications, deep biochemical and molecular biology approaches are needed to elucidate the interactions among bacteria and underlying surfaces. The understanding of the bacterial response and adaptation to nanostructures, along with the role of specific genetic pathways involved in the cell-to-cell communications (i.e., quorum sensing molecules) and biofilm formation, are the key issues to enable the development of nanosurfaces with active, smart and programmable antibacterial properties, also in view of recent findings disclosing novel ways of communication among bacteria based on natural nanotubes [13].

\section{Financial \& competing interests disclosure}

The authors have no relevant affiliations or financial involvement with any organization or entity with a financial interest in or financial conflict with the subject matter or materials discussed in the manuscript. This includes employment, consultancies, honoraria, stock ownership or options, expert testimony, grants or patents received or pending, or royalties.

No writing assistance was utilized in the production of this manuscript. 


\section{Bibliography}

1 Costerton JW, Stewart PS, Greenberg EP. Bacterial biofilms: a common cause of persistent infections. Science 284, 1318-1322 (1999).

2 Gristina AG. Biomaterial-centered infection: microbial adhesion versus tissue integration. Science 237, 1588-1595 (1987).

3 Mitragotri S, Lahann J. Physical approaches to biomaterial design. Nat. Mater. 8, 15-23 (2009).

4 Verma A, Uzun O, Hu Y et al. Surfacestructure-regulated cell-membrane penetration by monolayer-protected nanoparticles. Nat. Mater. 7, 588-595 (2008).

5 Rizzello L, Sorce B, Sabella S et al. Impact of nanoscale topography on genomics and proteomics of adherent bacteria. ACS Nano 5, 1865-1876 (2011).
6 Klemm P. Fimbriae Adhesion, Genetics, Biogenesis, and Vaccines. CRC Press, Boca Raton, FL, USA (1994).

7 Pouttu R, Puustinen T, Virkola R, Hacker J, Klemm P, Korhonen TK. Amino acid residue Ala-62 in the FimH fimbrial adhesin is critical for the adhesiveness of meningitisassociated Escherichia coli to collagens. Mol. Microbiol. 31, 1747-1757 (1999).

8 Blomfield IC. The regulation of pap and type 1 fimbriation in Escherichia coli. Adv. Microb. Physiol. 45, 1-49 (2001)

9 Bayer I, Fragouli D, Attanasio A et al. Water repellent cellulose fiber networks with multifunctional properties. ACS Appl. Mater. Interf. doi: 10.1021/am200891f (2011) (Epub ahead of print).

10 Kalyva M, Bertoni G, Milionis A, Cingolani R, Athanassiou A. Tuning of the characteristics of Au nanoparticles produced by solid target laser ablation into water by changing the irradiation parameters. Microsc. Res. Tech. 73, 937-943 (2010).

11 Matsumura Y, Yoshikata K, Kunisaki S, Tsuchido T. Mode of bactericidal action of silver zeolite and its comparison with that of silver nitrate. Appl. Environ. Microbiol. 69, 4278-4281 (2003).

12 Feng QL, Wu J, Chen GQ et al. A mechanistic study of the antibacterial effect of silver ions on Escherichia coli and Staphylococcus aureus. Biomed. Mater. Res. 52, 662-668 (2000).

13 Dubey GP, Ben-Yehuda S. Intercellular nanotubes mediate bacterial communication. Cell 144, 590-600 (2011).

\section{Patent}

101 Bayer I, Cingolani R, Athanassiou A. I0143202 (2010). 